\title{
Effect of crystallographic texture on the forming limit in microforming of brass
}

\author{
Anil Mashalkar* and Vilas Nandedkar \\ SGGS Institute of Engineering \& Technology, Nanded, India
}

Received: 7 July 2019 / Accepted: 7 October 2019

\begin{abstract}
Microforming is an emerging technology to manufacture products in the light of miniaturization in several domains of industry. Plastic anisotropy is one of the material characteristics significantly affecting the micro forming process. The crystal orientation influences tensile strength, yield strength and ductility, depending on different grain sizes and principle sliding planes. The present work elaborates on the influence of the plastic anisotropic in microforming for a plane stress state condition. Yield function and constitutive equations for the anisotropic material with consideration of the crystal lattice constants and parameters of crystallography texture are proposed. The crystal orientation is considered in a subroutine VUMAT algorithm, as an internal variable based on the developed mathematical model which is implemented in Abaqus as an user material subroutine. Micro limiting dome height experimental tests for different strain paths are conducted with brass foils. The results are compared with that predicted with numerical analysis, considering critical damage and element deletions. The numerical and experimental results show a good agreement for the Alpha brass ultrathin foils, using a set of failure criterion.
\end{abstract}

Keywords: Crystal orientation / stress-strain curve / anisotropy / forming limit / micro forming

\section{Introduction}

Advances in the electronics, mobiles, energy and medical applications are influenced by developments of new materials and their processing technologies. Due to miniaturization in many sectors, micro forming has emerged as a most preferred process for sheet metal components. The development of rational, science-based technology in the micro forming processes is primarily concerned with the need of the detailed study of material properties. One of the specific characteristics inherent in the majority of the material is anisotropy, which is based on crystallographic structure and texture formation under high plastic strain [1]. However, the assumption of the material isotropy is still being used in the finite element analysis, though it does not actually meet the real deformation condition. In the plasticity theory of the isotropic material, shifting from the elastic condition to the plastic is usually determined on the basis of the maximum shear stress criteria developed by maximum distortion strain energy criteria by Mises [2]. The major criterion does not consider the crystallographic texture of materials and consequently the anisotropy of their physical, mechanical and plastic properties.

\footnotetext{
* e-mail: anilmashalkar@gmail.com
}

It should be noted that the high accuracy of the recently proposed criterions is achieved by a large amount of the anisotropy coefficients (up to 18), determination of which involves numerous mechanical tests at different stress states [3]. Though the applied anisotropy coefficients characterize the anisotropy of plastic deformations, they do not take into account the reason for anisotropy, i.e., the crystallographic texture [4]. Thus, the mentioned yield functions, on one hand, allow describing the plastic flow of anisotropic materials. On the other hand, they do not allow carrying out technical analysis of micro thin foils considering the crystallographic texture [5]. As a result, it is difficult to determine the arrangement of crystallographic texture in terms of the necessities of specific microforming processes [6,7]. However, there is short of systematic research on the micro limiting dome height [LDH] test and failure mechanism of the ultra thin foils considering the crystal orientation. The effect of texture and grain structure on strain localisation and formability is investigated experimentally and numerically for two AlZnMg alloys [8] by Lademo et al. The strongly textured materials exhibit inferior formability to the materials with weak or nearly random texture. The reason for this is attributed to the reduced work-hardening capacity of the former materials and to a less degree to the plastic anisotropy. Grechnikov proposed calculation procedure 
which considers the crystal lattice constants and the parameters of crystallographic orientation of material. The main practical significance of this procedure is possibility to predict the effect of crystallographic texture of rolled sheets on limiting strains and formability of material in different metal forming process [9]. Masoud Hajian studied 1010 steel sheet formability. The initial texture of sheet material was measured through X-ray diffraction technique. Also, the stress-strain behaviour and FLD of the material were determined by performing simple tension and hemispherical punch tests, respectively. In order to predict the forming limits of the material by simulation, a UMAT subroutine was developed and linked to the non-linear finite element software ABAQUS. In this subroutine, a rate sensitive crystal plasticity model along with the power law hardening was implemented. Second-order derivative of sheet thickness variations with respect to time was used for necking criterion. The obtained FLD was compared with the experimental measurements and good agreement was found between simulation and experiment with acceptable errors between approximately 5-15\% [10]. María A. Bertinetti studied the effect of the cube texture on forming-limit strains is studied using a rate-dependent viscoplastic law in conjunction with the Marciniak-Kuczynski approach. The forming limit diagram and yield locus are determined for several spreading of grain orientations around the ideal $\{100\}\langle 001\rangle$ component [11]. Fulop et al. carried simulations of the mechanical response of ultra-thin ductile metal sheets. Rate-dependent single crystal plasticity theory was used to implement the algorithms into a Finite Element code. A uniaxial tensile test and a three-point bending test are computationally evaluated. The effect of the number of surface grains over the total number of grains is investigated numerically [12].

In this paper, the subroutine VUMAT is applied with the plasticity criteria and crystallographic orientation. Crystal lattice constants and crystallographic orientation parameters are included explicitly and implemented in numerical analysis of the micro-LDH test considering the orientation of the blanks. Also, study involves investigations on influence of crystallographic orientation on formability in microforming process and outcomes of numerical approach are validated with the experimental results.

\section{Limiting dome height test}

Micro-formability of metal foils can be measured using LDH Test. Specimen for the tensile test were designed as per ASTM E 345 standard and the properties achieved from various tests are presented in Table 1. These specimen are designed such that, the strain paths can be achieved in both drawing as well as stretching zones using uniaxial strain, plane strain and biaxial strain. Figure 1 shows the FSA M100 universal testing machine of capacity $100 \mathrm{KN}$ with micro limit height dome attachment. Experiments were performed for the ultra thin foil of $40 \mu \mathrm{m}$ thickness of alpha brass material and Figure 2 shows the micro-formed sample.
Table 1. Properties of brass.

\begin{tabular}{ll}
\hline Properties & Value \\
\hline Young's modulus $(\mathrm{e})=\operatorname{props}(1)$ & $6255 \mathrm{MPa}$ \\
xnu $=\operatorname{props}(2)$ & 0.29 \\
Yield stress $=\operatorname{props}(3)$ & $296 \mathrm{MPa}$ \\
Plastic slope $($ hard $)=\operatorname{props}(4)$ & $1425 \mathrm{MPa}$ \\
Hardening coeff $(n)=\operatorname{props}(5)$ & 0.34 \\
Yield strain $($ eyield $)=\operatorname{props}(9)$ & 0.06 \\
\hline
\end{tabular}

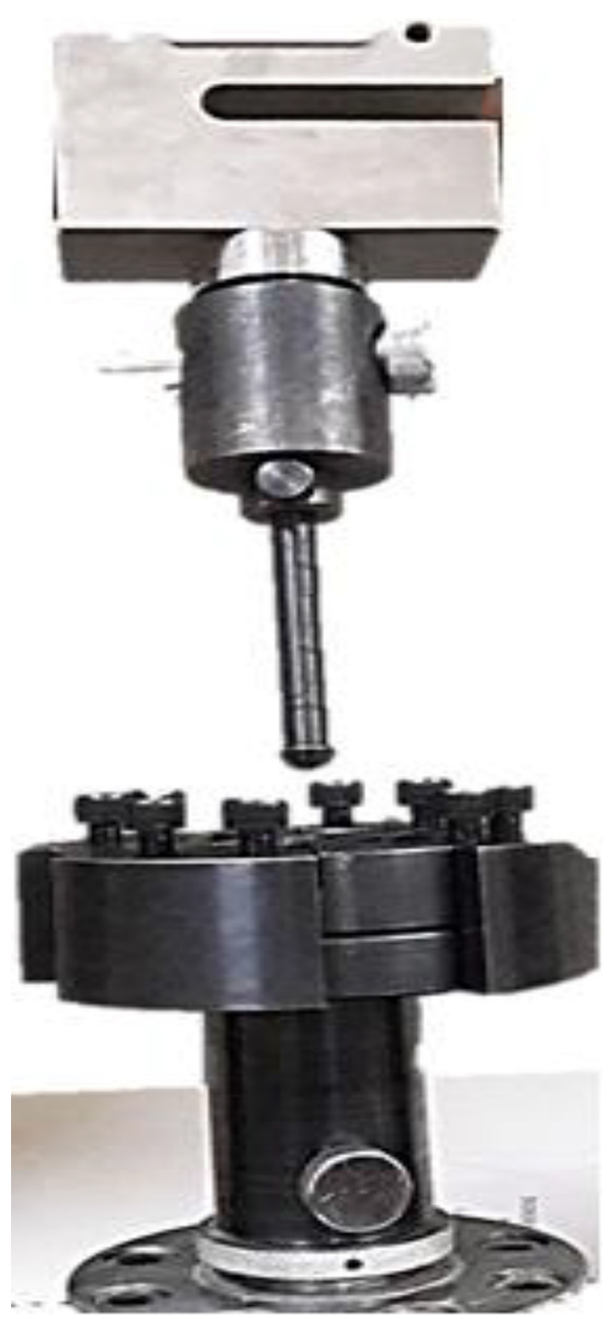

Fig. 1. LDH experimental setup.

\section{True strain measurement}

Circle grid with diameter of one mm each, and center to center distance of $2 \mathrm{~mm}$ were printed on specimen to measure deformations. An optical microscope was used to record the minor and major axis of the ellipse. The equations for percentage true major and minor strain is 


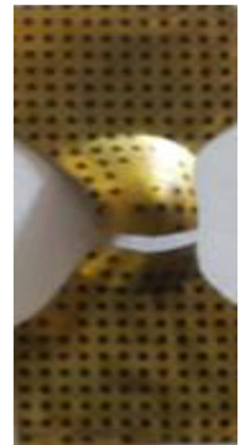

Uniaxial Strain Paths

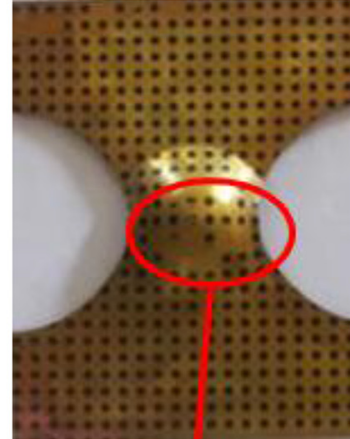

Biaxial Strain Paths

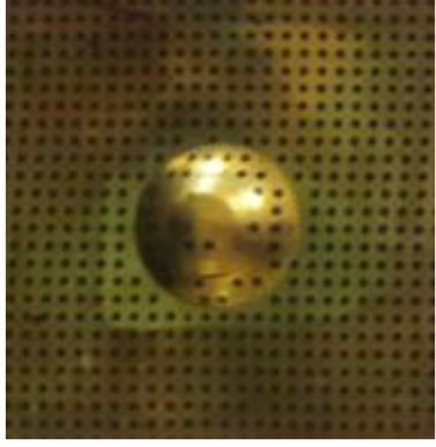

Plane Strain Paths

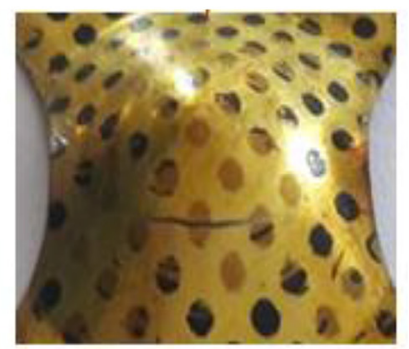

Fig. 2. Micro-formed alpha brass ultra-thin foil.

given in equations (1) and (2):

$$
\begin{aligned}
& \text { major train }=\frac{\text { Instanteneous Major Axis Length }}{\text { Original Circle Diameter }} \times 100 . \\
& \text { minor strain }=\frac{\text { Instanteneous Minor Axis Length }}{\text { Original Circle Diameter }} \times 100 .
\end{aligned}
$$

Uniaxial strain, plane strain and biaxial strains for $40 \mu \mathrm{m}$ brass foil were measured from experimentation as presented in Table 2. Forming limit curves has been plotted using these values.

\section{Finite element model of the micro LDH test using vumat subroutine}

Figure 3 shows the numerical model of the micro LDH test. The dimensions and geometry of the model corresponds to the standard test for stretch forming. In all cases, the blank thickness is $40 \mu \mathrm{m}$. The finite element model has been discretized using S3R and S4R shell element with reduced integration point over the thickness, in order to reduce the number of elements. S4R is the linear, finitemembrane-strain, quadrilateral shell element and is robust in nature. S3R is the linear, finite-membranestrain, triangular shell element applied to capture bending deformations or high strain gradients because of the constant strain approximation in the elements. The
Table 2. Maximum in-plane and minimum in-plane principal strain values from the physical experimentation.

\begin{tabular}{llll}
\hline Thickness & Specimen & Major strain & Minor strain \\
\hline $40 \mu \mathrm{m}$ & Uniaxial & 0.263 & -0.123 \\
& Plane & 0.250 & 0.015 \\
& Biaxial & 0.273 & 0.170 \\
\hline
\end{tabular}

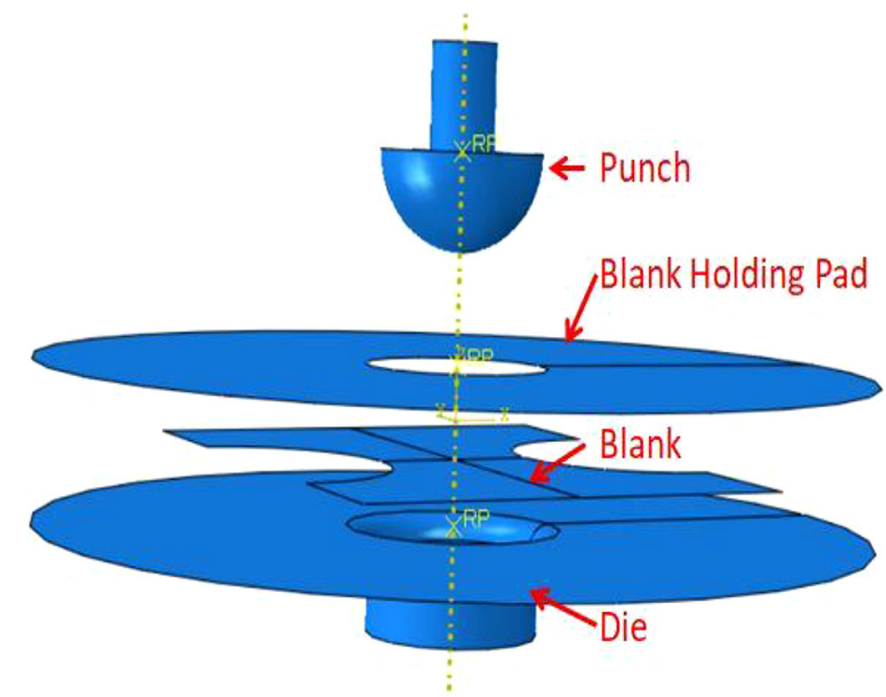

Fig. 3. Modeling of the process tool with Abaqus for LDH. 
Table 3. Crystallographic orientations and their parameters.

\begin{tabular}{lllll}
\hline & Orientation & & \multicolumn{3}{c}{ Generalized anisotropy parameters } \\
\cline { 3 - 5 } Name & Designation & & $\eta_{12}$ & $\eta_{23}$ \\
\hline Copper & $\{112\}\langle 111\rangle$ & 0.490 & 1.127 & 0.490 \\
Brass & $\{110\}\langle 112\rangle$ & 0.490 & 0.490 & 1.127 \\
S & $\{123\}\langle 634\rangle$ & 0.575 & 0.808 & 0.808 \\
Rotated cube & $\{100\}\langle 011\rangle$ & 0.147 & 1.764 & 1.764 \\
Cube & $\{100\}\langle 001\rangle$ & 1.764 & 1.764 & 1.764 \\
Goss & $\{110\}\langle 001\rangle$ & 1.764 & 0.147 & 1.764 \\
Isotropy & & 1 & 1 & 1 \\
\hline
\end{tabular}

combination of these two will ensure complete discretization of the model and capture all deformations with minimal error. Between the tool and blank contact, pairs were prescribed, and the friction obeys the Coulombs law. The tool was assumed absolutely to be rigid. The model of anisotropic elasto-plastic material considering crystallographic texture is used to describe the blank material behavior of alpha brass. In order to assess the influences of crystallographic structure on the formability, it was modeled anisotropic material, the texture of which is represented by crystallographic orientation. The characteristic for the rolled material deformation orientation of brass is $\{110\}\langle 112\rangle$, rotated cube $\{100\}\langle 011\rangle$, cube $\{100\}$ $\langle 001\rangle$ and isotropy. The orientation parameters are given in Table 3 [13].

\section{Continuum damage model}

Damage is addressed as one of the output measure and its evolution law is given as a general function of other state variables such as stress, plastic strain, temperature and so on. From a general point of view, the damage variable should be described using a tensor formulation [14]. From the physical point of view, damage variable indicates the progressive material deterioration due to non-reversible deformation processes and can be expressed by the reduction of the nominal section area of a given reference volume element (RVE) as a result of micro-voids formation and growth. Let's consider the damage due to growth of micro-cavities, atomic bond breaking, discontinuous surface.

$$
\text { Damage }[D]=\frac{A_{D}}{A}
$$

where $A$ - overall area of the damage body, $A_{D}$ - damaged area.

Therefore, $D$ is scalar and values between 0 and 1

$$
\begin{gathered}
\bar{\sigma}=\frac{F}{\left(A-A_{D}\right)} . \\
\bar{\sigma}=\frac{F}{A\left(1-A_{D} / A\right)}
\end{gathered}
$$

where $D$ is the overall damage variable and $\bar{\sigma}$ is the effective (or undamaged) stress tensor computed in the current increment. $\bar{\sigma}$ are the stresses that would exist in the material in the absence of damage. The material has lost its load-carrying capacity when $D=1$.

$$
\bar{\sigma}=\frac{\sigma}{(1-D)} .
$$

True stress was replaced by effective stress [15]

$$
\sigma=E_{0}(1-D) \varepsilon
$$

\section{Damage evolution}

Based on Swift's law [16] the damage evolution equation is

$$
\sigma_{y}=K\left(\varepsilon_{0}+p\right)^{n}
$$

$$
\begin{aligned}
\operatorname{Damage}(D) & =\left(\frac{D_{C}}{\varepsilon_{\mathrm{PR}}-\varepsilon_{\mathrm{PD}}}\right) \\
& \times\left[\frac{2}{3}(1-v)+3(1-2 v)\left(\frac{\sigma_{H}}{\sigma_{e q}}\right)\right]\left(\varepsilon_{0}+p\right)^{2 n} p
\end{aligned}
$$

where $\varepsilon_{\mathrm{PD}}$, plastic strain under which the damage evolution is negligible; $\varepsilon_{\mathrm{PR}}$, plastic strain at rupture; $D c$, damage parameter at rupture called the critical value of the damage; $\sigma_{H}$, hydrostatic stress; $\sigma_{\text {eq }}$, equivalent stress; $\sigma_{y}$, yield stress; $\varepsilon_{0}$, strain value at yield; $K$ and $n$ are isotropic hardening coefficients; $p$, equivalent plastic strain.

$\sigma_{e q}=\sqrt{\frac{\eta_{12+} \eta_{31}}{2} \cdot \sigma_{12}^{2}+\frac{\eta_{12+} \eta_{23}}{2} \cdot \sigma_{22}^{2}-\eta_{12} \sigma_{11} \sigma_{22}+\left(5-2 \eta_{12}\right) \sigma_{12}^{2}}$.

Here $\sigma_{i j}$ is stress tensor [4]. The generalized anisotropic parameters $\eta_{i j}$ are defined by

$$
\eta_{i j}=1-\frac{15\left(A^{l}-1\right)}{3+2 A^{l}}\left(\Delta_{i}+\Delta_{j}-\Delta_{k}-\frac{1}{5}\right),
$$


where $A^{1}$ is the anisotropic parameter of the crystal lattice $\Delta_{i}$. Are the orientation factor of crystallographic orientation.

$$
\Delta_{i}=\frac{h_{i}^{2} k_{i}^{2}+k_{i}^{2} l_{i}^{2}+l_{i}^{2} h_{i}^{2}}{\left(h_{i}^{2}+l_{i}^{2}+k_{i}^{2}\right)^{2}},
$$

$h_{i}, l_{i}, k_{i}$ are Miller indices defining the eighth direction in crystal with respect to a coordinate system associated with the blank. The rolling direction set along the $x$-axis.

Strain tensor for 3D is written as

$$
p=\sqrt{\frac{2}{3} \varepsilon^{p}: \varepsilon^{p}} .
$$

Strain equivalence principle yielding occurs

$$
\tilde{\sigma}=\frac{\sigma}{1-D}=\sigma_{y}=K\left(\varepsilon_{0}+p\right)^{n}
$$

After yielding, plastic deformation occurs due to the deviatoric stress, which consists of unequal principalstresses. Deviatoric stress is the difference between principle stress and hydrostatic stress. Another way of representative linear elastic stress-strain is given below

$$
\sigma=\lambda \operatorname{trace}(\varepsilon) I+2 \mu \varepsilon
$$

The stress equation including the damage can be written as

$$
\sigma=(1-D)(\lambda \operatorname{trace}(\varepsilon) I+2 \mu \varepsilon) .
$$

Initially, the time step is zero and at first increment the new trial stress tensor is evaluated from the linear stress strain curve. If the new calculated trial stress value is less than the yield stress, then it is stored as new trial stress value. When the trial stress exceeds yield point and enters plastic region, damage criterion is applied, so new trail stress and plastic strain is due to damage. Also, plastic strain and yield stress values are updated and tested for the damage initiation. Crack initiation is considered when $D$ value exceeds $0.9 . \Delta D$ is calculated when $p>\varepsilon_{\mathrm{PD}} \Delta \gamma$ calculated from the below given equations. $\Delta$ is determined considering constant $D$ during one step which is justified in the explicit calculation because of very small increments

$$
\Delta \gamma=\frac{\sqrt{\sigma_{\text {new }}^{\text {Dtrial }}: \sigma_{\text {new }}^{\text {Dtrial }}}-\sqrt{\frac{2}{3}}\left(1-D_{\text {old }}\right) \sigma_{\text {yold }}}{2 \mu\left(1+\frac{h}{3 \mu}\right)\left(1-D_{\text {old }}\right)}
$$

$h$, isotropic hardening law.

\section{Results and discussion}

Limiting Dome Height test for $40 \mu \mathrm{m}$ alpha brass foil was carried out numerically with ABAQUS using VUMAT subroutine and the results of maximum in-plane principle strain and minimum in-plane principle strain in three cases uniaxial strain, plane strain and biaxial strain are plotted below for the different orientation (orientation of brass $\{110\}\langle 112\rangle$, Rotated cube $\{100\}\langle 011\rangle$, Cube $\{100\}\langle 001\rangle$ and Isotropy).

\subsection{LDH test for $\{110\}\langle 112\rangle$}

The results for LDH test for $\{110\}\langle 112\rangle$ are plotted below in Figures 4-6.

From the graphs it is evident that fracture for maximum principle strain in uniaxial stretching occurs at 0.16 , plane strain at 0.24 and in biaxial at 0.32 . Maximum in-plane strain shown by average value nearer to red zone elements in Figures 4a, 5a and 6a. In case of minor principle strain necking occurs at -0.1 in uniaxial stretching, 0.015 in plane stretching and at 0.17 in biaxial stretching shown by average nearer to brown zone elements in Figures 4b, 5b and 6b.

\subsection{LDH test for $\{100\}\langle 011\rangle$}

Results of limiting dome height test $\{100\}\langle 011\rangle$ for $40 \mu \mathrm{m}$ alpha brass foil are plotted below in Figures $7-9$. From the numerical outcomes it is evident that fracture for maximum principle strain in uniaxial stretching occurs at 0.12 , plane strain at 0.14 and in biaxial at 0.35 . Maximum in-plane strain shown by average value nearer to red zone elements in Figures 7a, 8a and 9a. In case of minor principle strain necking occurs at -0.06 in uniaxial

\section{Orientation}

Brass

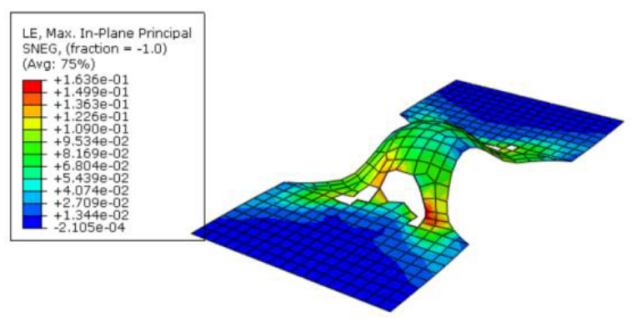

(a)

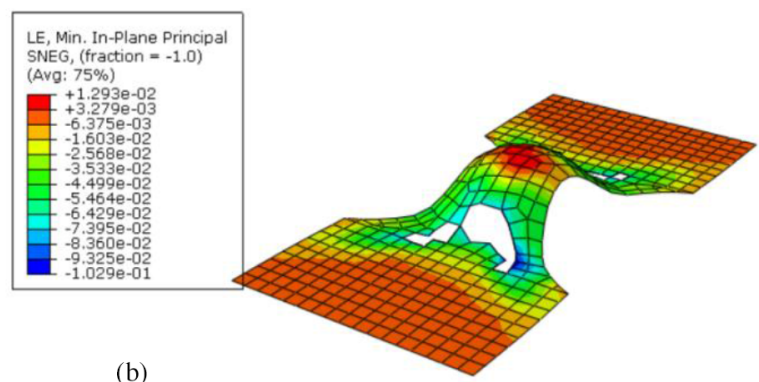

(b)

Fig. 4. (a) Maximum in-plane principle strain under uniaxial stretching. (b) Minimum in-plane principle strain under uniaxial stretching. 
Brass
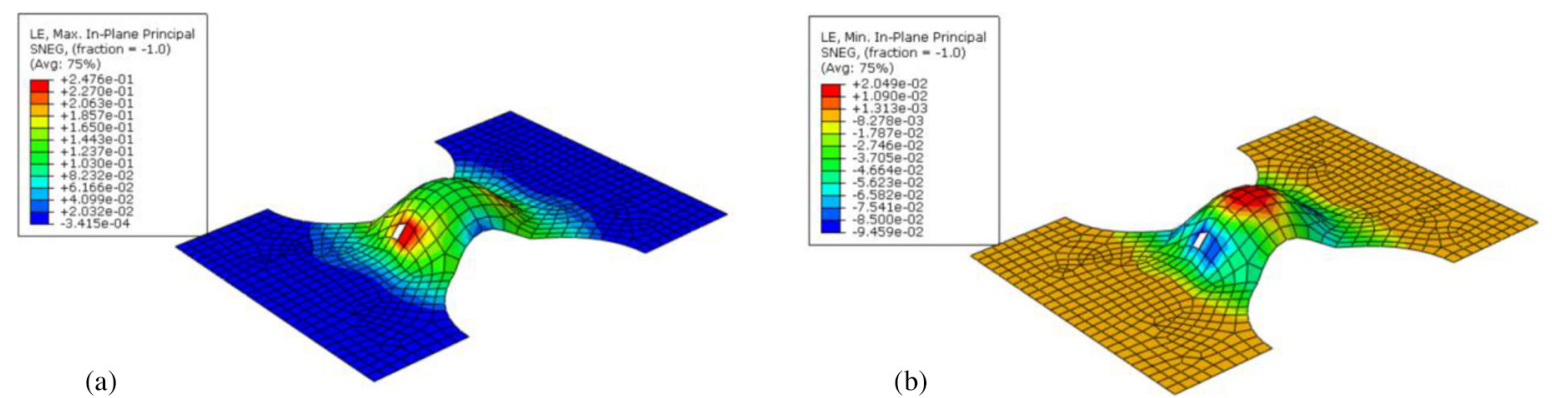

Fig. 5. (a) Maximum in-plane principle strain under plane stretching. (b) Minimum in-plane principle strain under plane stretching.

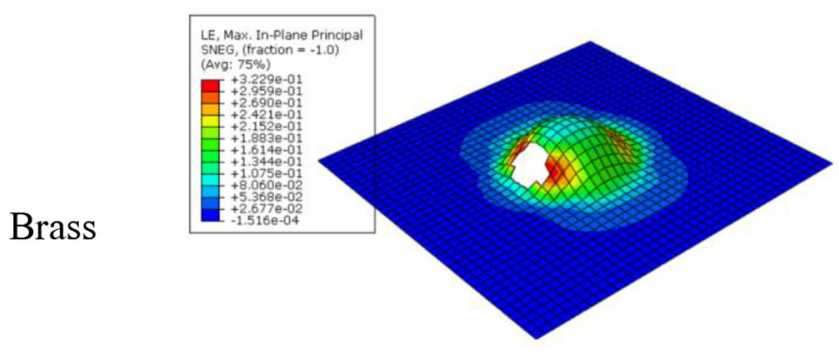

(a)

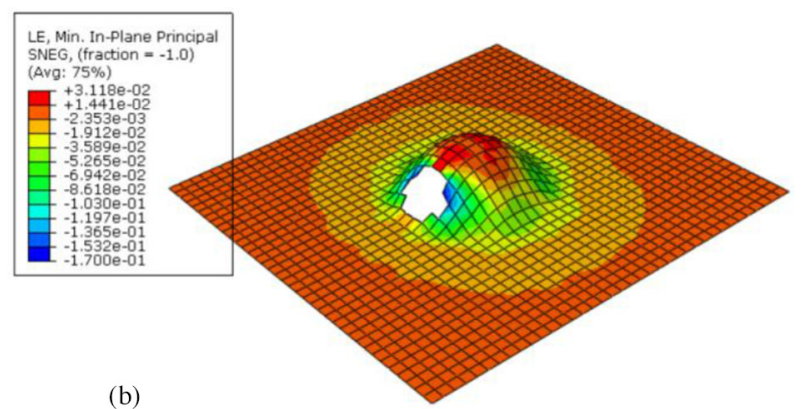

Fig. 6. (a) Maximum in-plane principle strain under biaxial stretching. (b) Minimum in-plane principle strain under biaxial stretching.

Cube

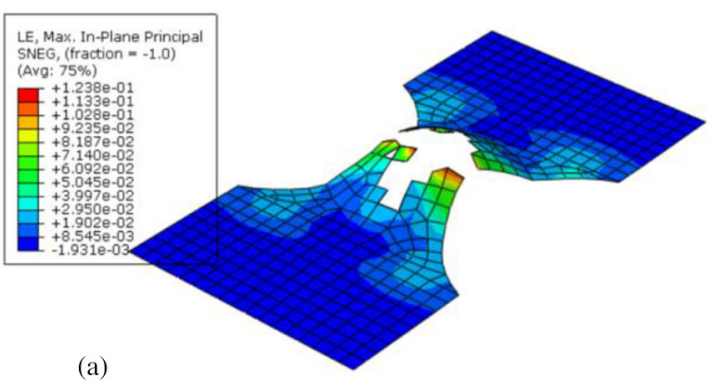

(a)

Fig. 7. (a) Maximum in-plane principle strain under uniaxial stretching. stretching.

stretching, -0.001 in plane stretching and at 0.1465 in biaxial stretching shown by average nearer to brown zone elements in Figures 7b, 8b and 9b.

\subsection{LDH test for $\{100\}\langle 001\rangle$}

Results pertaining to limiting dome height test $\{100\}\langle 001\rangle$ are presented in Figures 10-12. It is evident that facuture for maximum principle strain in uniaxial stretching occurs at 0.23 , plane strain at 0.17 and in biaxial at 0.32 . Maximum in-plane strain shown by average value nearer to red zone elements in Figures 10a, 11a and 12a. In case of minor principle strain necking occurs at -0.13 in uniaxial stretching, 0.015 in plane stretching and at 0.1368 in biaxial stretching shown by average nearer to brown zone elements in Figures 10b, 11b and 12b.

\subsection{LDH test for isotropy}

Limiting Dome Height testisotropy for $40 \mu \mathrm{m}$ alpha brass foil was carried out numerically with ABAQUS and the results of maximum in-plane strain and minmum in-plane strain for three strain path cases uniaxial strain, plane strain and biaxial strain are plotted below in Figures 13-15. From the graphs it is evident that fracture for maximum principle strain in uniaxial stretching occurs at 0.1238 , plane strain at 0.1466 and in biaxial at 0.3511 . Maximum in-plane strain shown by average value nearer to red zone elements in Figures 13a, 14a and 15a. In case of minor principle strain necking occurs at -0.081 in uniaxial stretching, -0.01 in plane stretching and at 0.17 in biaxial stretching shown by average nearer to brown zone elements in Figures 13b, 14b and 15b. 


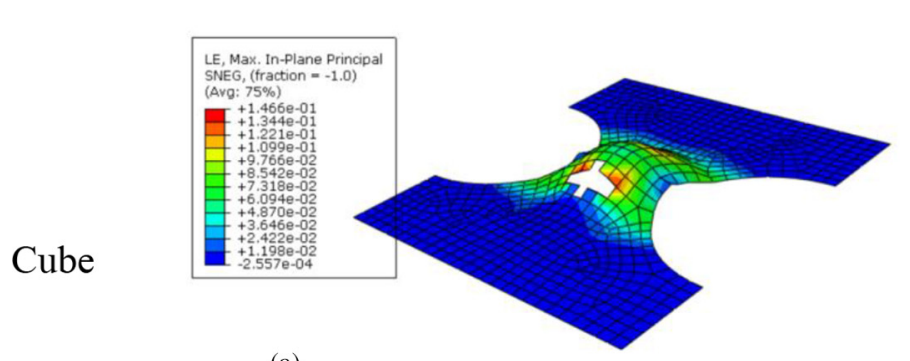

(a)

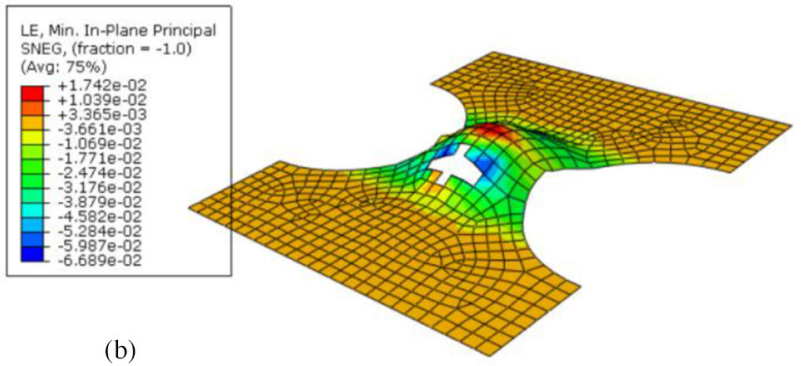

(b)

Fig. 8. (a) Maximum in-plane principle strain under plane stretching. (b) Minimum in-plane principle strain under plane stretching.

\section{Cube}

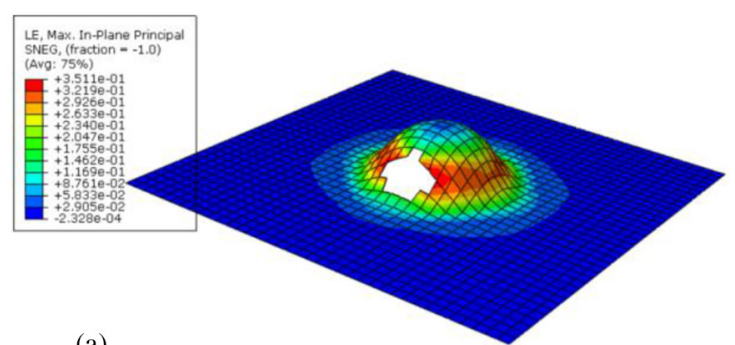

(a)

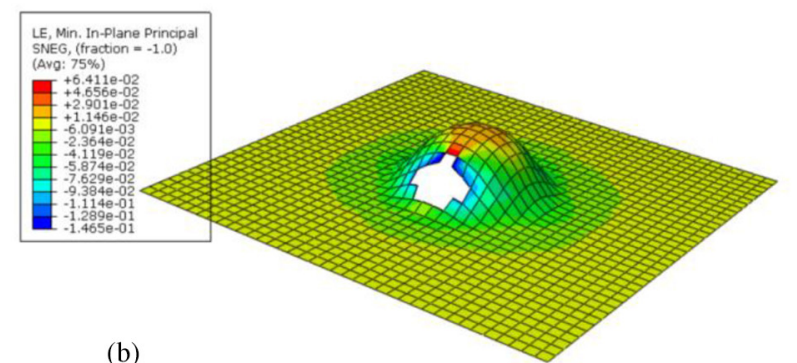

(b)

Fig. 9. (a) Maximum in-plane principle strain under biaxial stretching. (b) Minimum in-plane principle strain under biaxial stretching.

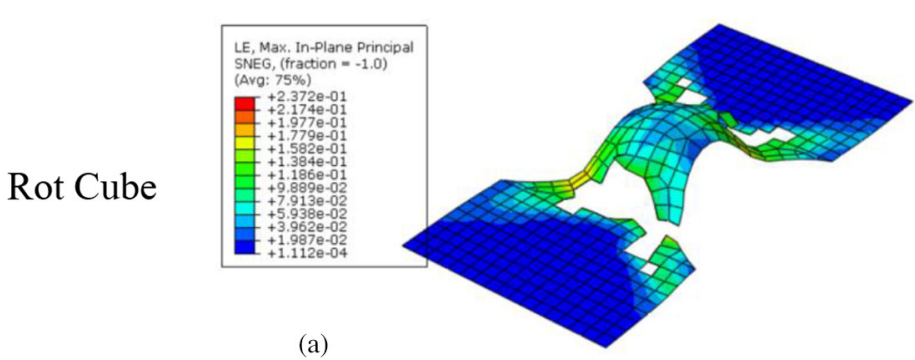

(a)

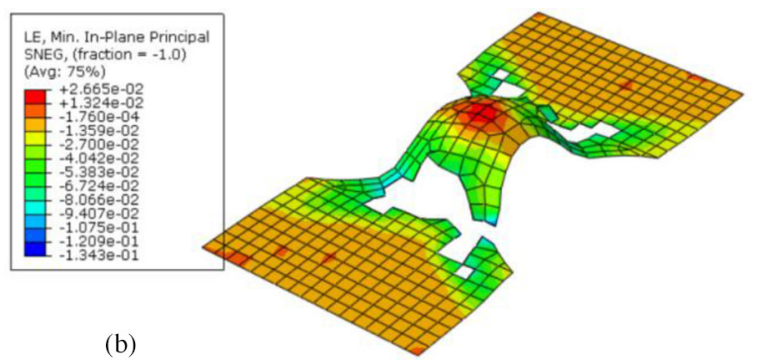

(b)

Fig. 10. (a) Maximum in-plane principle strain under uniaxial stretching. (b) Minimum in-plane principle strain under uniaxial stretching.

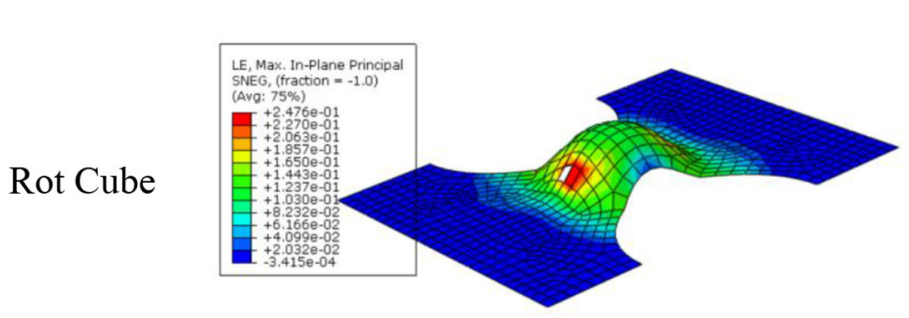

(a)

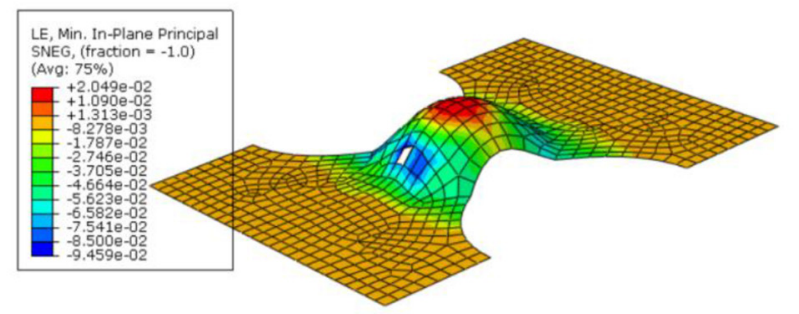

(b)

Fig. 11. (a) Maximum in-plane principle strain under plane stretching. (b) Minimum in-plane principle strain under plane stretching. 


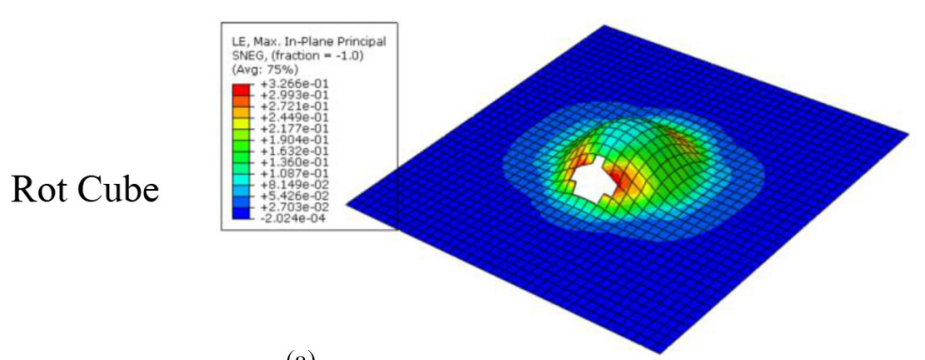

(a)

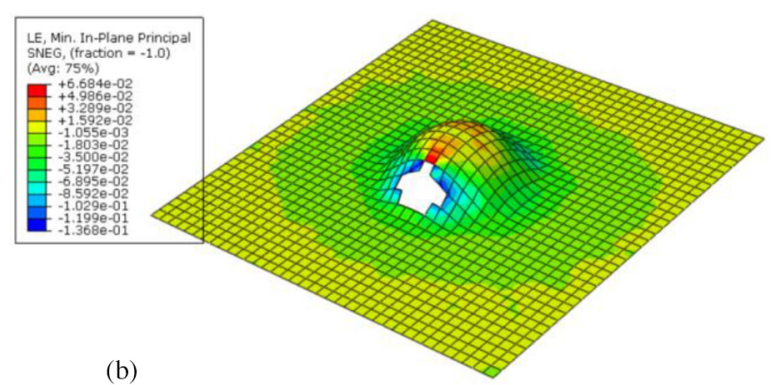

(b)

Fig. 12. (a) Maximum in-plane principle strain under biaxial stretching. (b) Minimum in-plane principle strain under biaxial stretching.

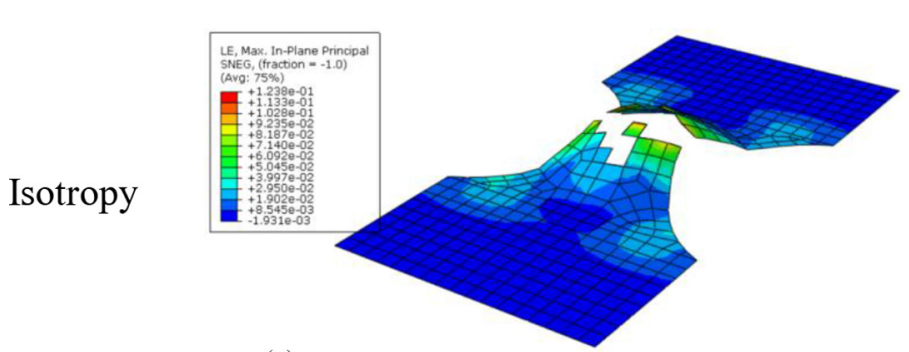

(a)

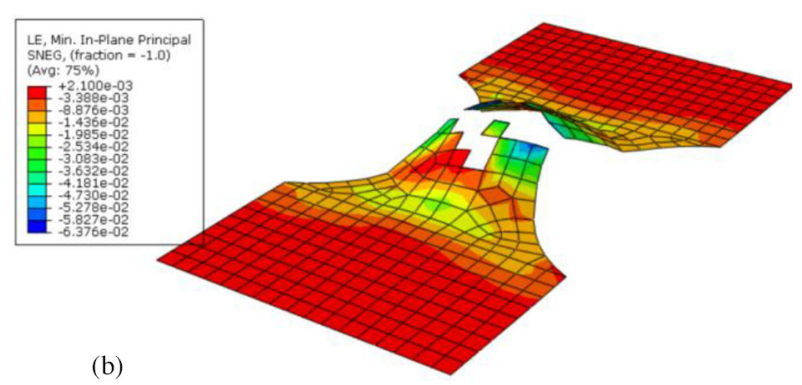

Fig. 13. (a) Maximum in-plane principle strain under uniaxial stretching. (b) Minimum in-plane principle strain under uniaxial stretching.
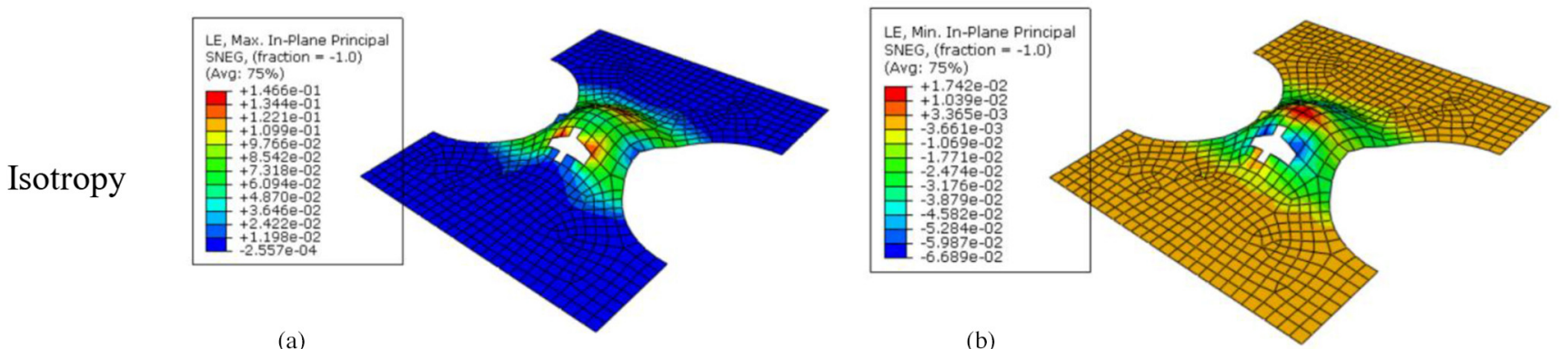

Fig. 14. (a) Maximum in-plane principle strain under plane stretching. (b) Minimum in-plane principle strain under plane stretching.

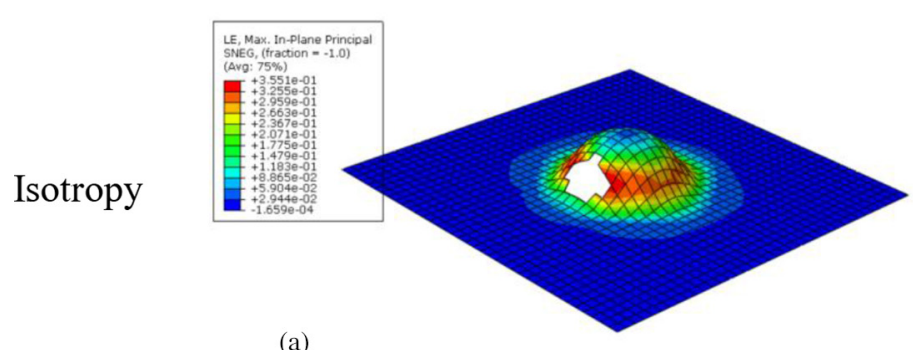

(a)

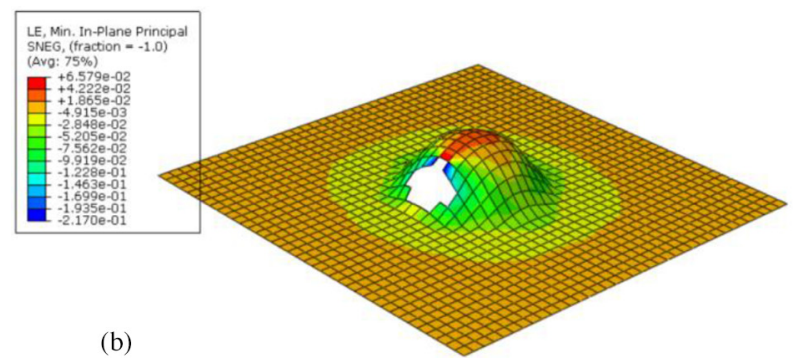

(b)

Fig. 15. (a) Maximum in-plane principle strain under biaxial stretching. (b) Minimum in-plane principle strain under biaxial stretching. 
Table 4. Maximum in-plane and minimum in-plane principal strain values from the numerical simulation.

\begin{tabular}{|c|c|c|c|c|c|c|c|c|}
\hline \multirow[t]{2}{*}{ Description } & \multicolumn{2}{|c|}{ Brass } & \multicolumn{2}{|c|}{ Rotated cube } & \multicolumn{2}{|c|}{ Cube } & \multicolumn{2}{|c|}{ Isotropy } \\
\hline & $\begin{array}{l}\text { Minor } \\
\text { strain }\end{array}$ & $\begin{array}{l}\text { Major } \\
\text { strain }\end{array}$ & $\begin{array}{l}\text { Minor } \\
\text { strain }\end{array}$ & $\begin{array}{l}\text { Major } \\
\text { strain }\end{array}$ & $\begin{array}{l}\text { Minor } \\
\text { strain }\end{array}$ & $\begin{array}{l}\text { Major } \\
\text { strain }\end{array}$ & $\begin{array}{l}\text { Minor } \\
\text { strain }\end{array}$ & $\begin{array}{l}\text { Major } \\
\text { strain }\end{array}$ \\
\hline Uniaxial & -0.1 & 0.16 & -0.1343 & 0.2372 & -0.06376 & 0.1238 & -0.081 & 0.1238 \\
\hline Plane & 0.015 & 0.2476 & 0.015 & 0.1742 & -0.001 & 0.1466 & -0.01 & 0.1466 \\
\hline Biaxial & 0.17 & 0.322 & 0.1368 & 0.3266 & 0.1465 & 0.3511 & 0.17 & 0.355 \\
\hline
\end{tabular}

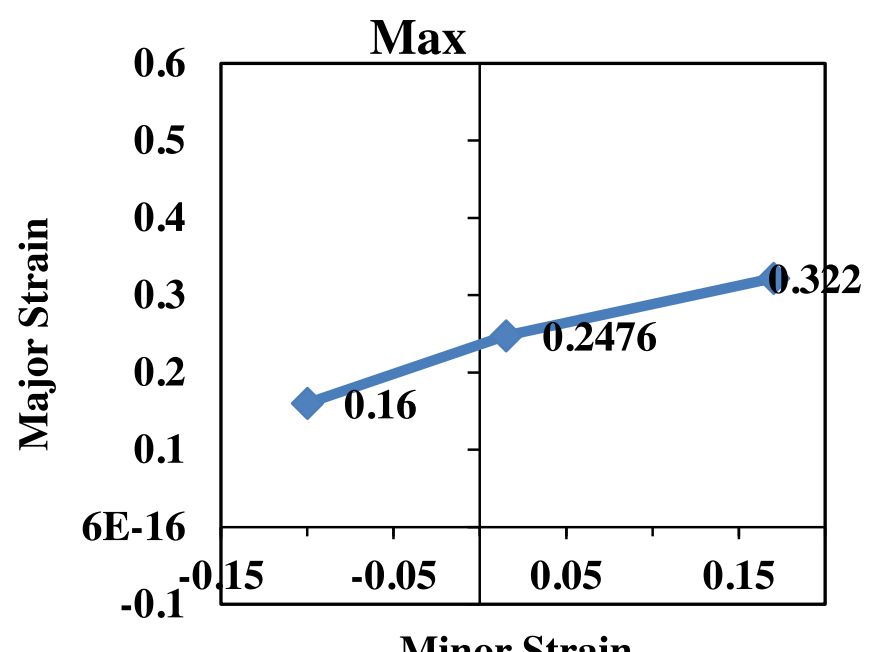

Fig. 16. Forming limit diagram for brass orientation numerical simulation.

\subsection{Forming limit curves (FLC)}

For plotting FLC maximum values of the major and the minor strain are determined by measuring the principal strains at failure state. Numerical simulation of failure limit curves is carried out using finite element analysis platform ABAQUS.

The details of maximum in-plane principle strain and minimum in-plane principle strain at different strain paths for thin foil (uniaxial, plane and biaxial) are presented in Table 4. For the construction of the forming limit diagram (FLD) maximum in-plane principle strain is plotted on $\mathrm{Y}$ axis and minimum in-plane principle strain on $x$-axis. The forming limit curves were plotted by joining limit strain co-ordinates and this procedure was repeated for all four crystallographic orientations. Numerically plotted FLDs are shown in Figures 16-19. It is apparent from Figures 16-19 that, as the orientation of foil changes forming limit curve changes. The area below curve presents safe zone for forming. Higher the curve on major stain axis, higher the formability. Amongst four cases investigated Brass orientation has higher formability, rotated cube is at second place, cube and isotropy orientation has almost same formability as curve resembles. Cube and isotropy orientation gives the same values of the fracture strain.

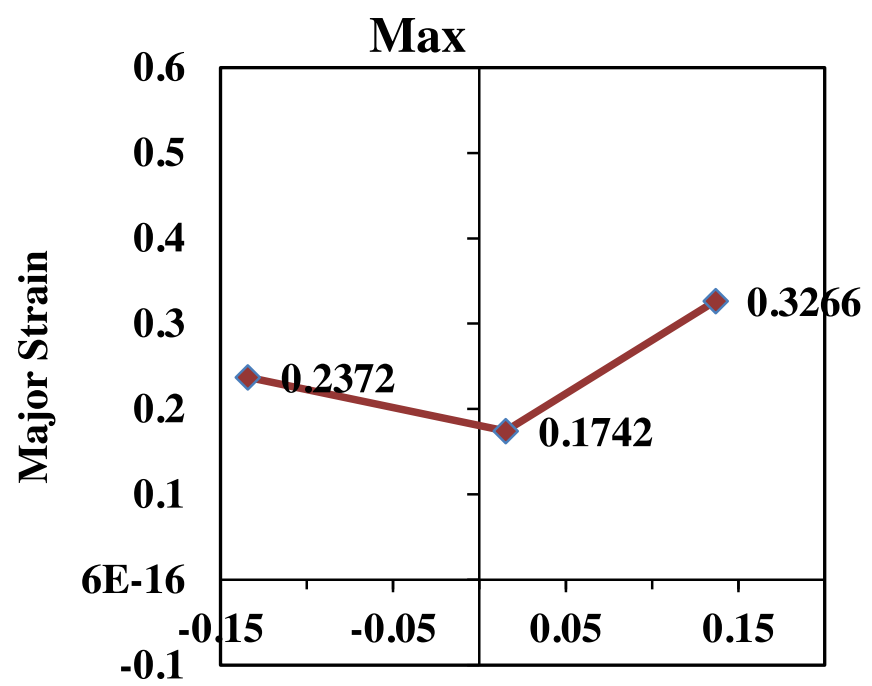

Minor Strain

Fig. 17. Forming limit diagram for rotated cube - numerical simulation.

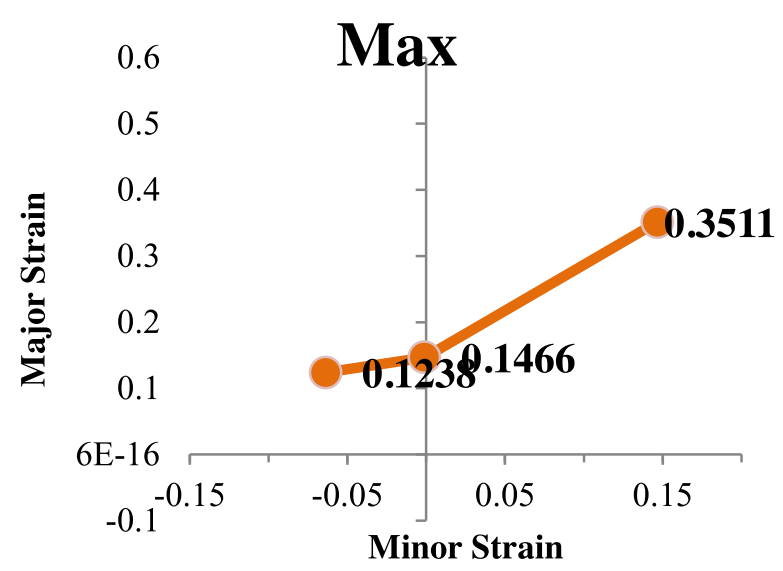

Fig. 18. Forming limit diagram for cube - numerical simulation.

\subsection{Validation of numerical results with experimental test data}

For $\{110\}\langle 112\rangle$ orientation, experimental and numerical failure limit curves are presented below in Figure 20. It is observed that maximum uniaxial strain in both approaches 


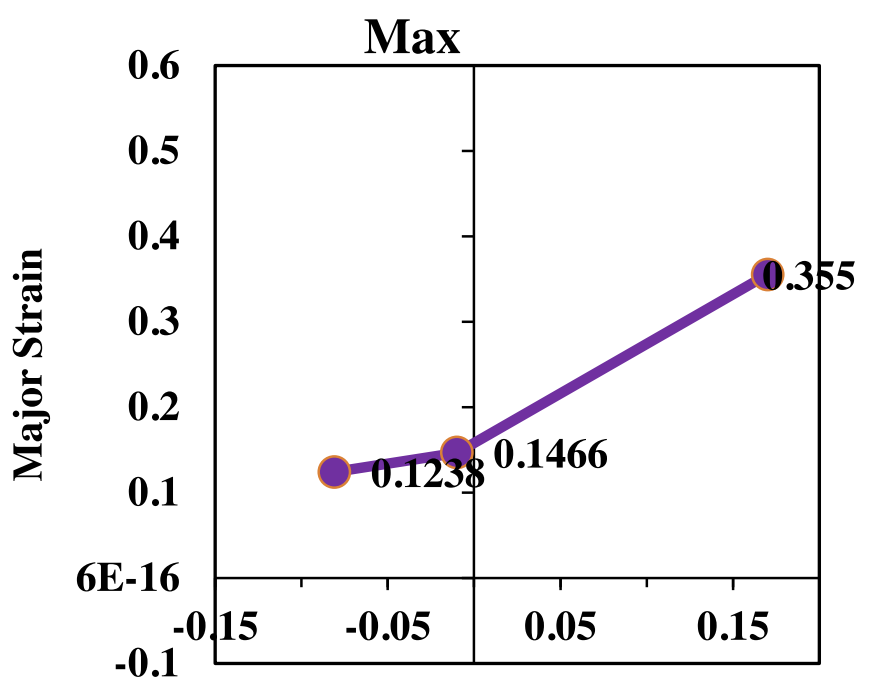

Minor Strain

Fig. 19. Forming limit diagram for isotropy - numerical simulation.

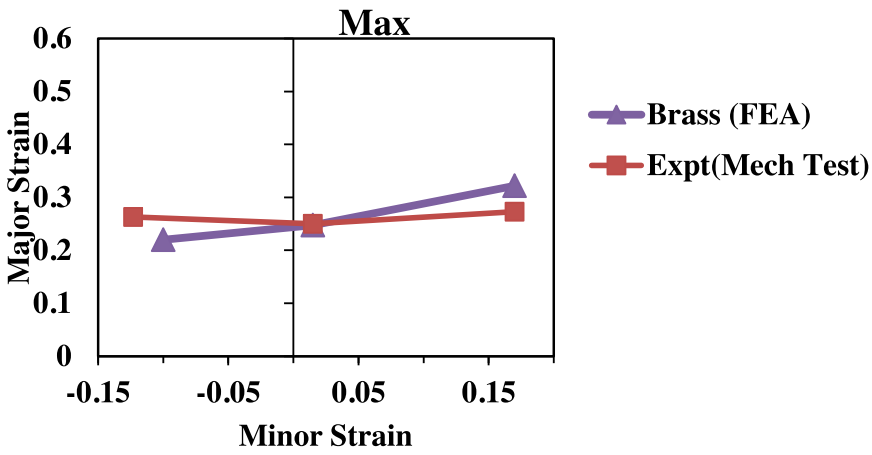

Fig. 20. Comparison of numerical and experimental failure limit curves for brass orientation.

is in full agreement within $20 \%$ error. Maximum plain strain is almost same in numerical approach with that of experimental. The state of maximum biaxial strain in both approaches is within $18 \%$ error.

When plotted both the failure limit curves as shown below they are in found to be of the same nature. It is obvious that numerical results strongly agree with experiment. For $\{100\}\langle 001\rangle$ orientation, experimental and numerical failure limit curves are presented below in Figure 21. It is observed that maximum uniaxial strain in both approaches is in full agreement within $10 \%$ error. Maximum plain strain within $20 \%$ error in numerical approach with that of experimental. The state of maximum biaxial strain is in both approaches is in then $20 \%$ error. When plotted both the failure limit curves as shown below they are in found to be of the same nature. It is obvious that numerical results strongly agree with experiment. Other orientation given more than $20 \%$ error.

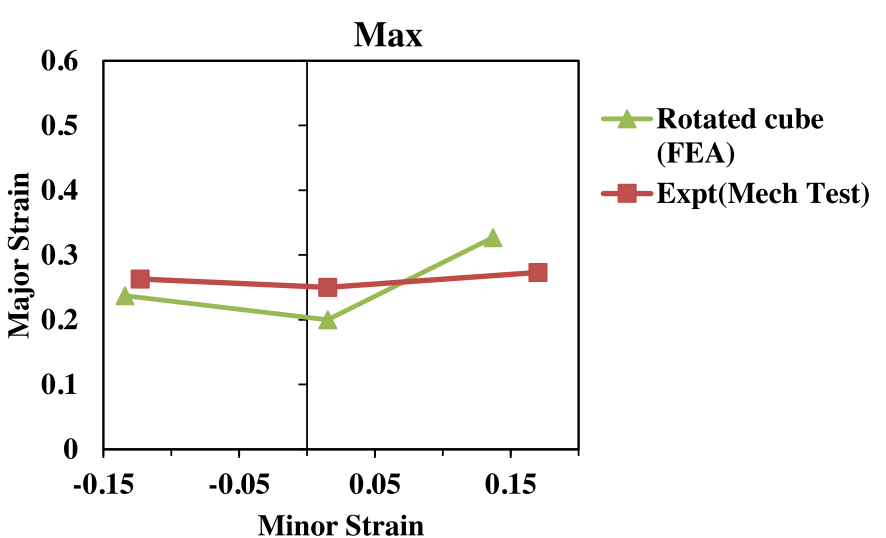

Fig. 21. Comparison of numerical and experimental failure limit curves for rotated cube orientation.

\section{Conclusions}

Based on the numerical simulations and experiments on the study of the forming limit of microforming of brass, the following conclusions can be drawn:

- The Brass orientation and rotating cube orientation results match with the experimental results within $20 \%$ error, and they show that the material has crystallographic orientation of brass and rotating cube orientation texture for the alpha brass C26000.

- The simulation results relating to the isotropy do not match experimental results well, which means that the material obeys the anisotropic material behavior.

- The results show limiting values in the FLD diagram for the different orientations, which will help the process and tool designs taking into account of the crystallographic orientation effects.

\section{References}

1. W. Truszkowski, The Plastic Anisotropy in Single Crystals and Polycrystalline Metals, Springer, Netherlands, 2001

2. S. Soare, D. Banabic, About mechanical data required to describe the anisotropy of thin sheets to correctly predict the earning of deep-drawn cups, Int. J. Plasticity 4 (2008) 34-37

3. R. Mises, Mechanics of Solid Bodies in the PlasticallyDeformable State, Nachr. d. Kgl. Ges. Wiss. Göttingen, Math.-phys. Klasse 1 (1913) 582-592

4. Y.A. Erisov, F.V. Grechnikov, S.V. Surudin, Yield function of the orthotropic material considering the crystallographic texture, Struct. Eng. Mech. 58 (2016) 677-687

5. F.V. Grechnikov, Y.A. Erisov, Virtual material model with the given crystallographic orientation of the structure, Key Eng. Mater. 684 (2016) 134-142

6. Y. Erisov, F. Grechnikov, Proceeding of 2015 International Conference on Mechanical Engineering, Automation and Control Systems, MEACS, 2015

7. F.V. Grechnikov, Y.A. Erisov, S. Alexandrov, Effect of anisotropic yield criterion on the springback in plane strain pure bending, in CEUR Work-shop Proceedings, 2016, pp. $569-577$ 
8. O.G. Lademo, K.O. Pedersen, T. Berstad, T. Furu, O.S. Hopperstad, An experimental and numerical study on the formability of textured AlZnMg alloys, Eur. J. Mech. A 27 (2008) 116-140

9. F.V. Grechnikov, S.V. Surudin, Ya.A. Erisov, A.O. Kuzin, I.N. Bobrovskiy, Influence of material structure crystallography on its formability in sheet metal forming processes, IOP Conf. Ser.: Mater. Sci. Eng. 286 (2017) 012021

10. M. Hajian, A. Assempour, Experimental and numerical determination of forming limit diagram for 1010 steel sheet: a crystal plasticity approach, Int. J. Adv. Manuf. Technol. 76 (2019) 1757-1767

11. M.A. Bertinetti, P.A. Turner, J.W. Signorelli, Investigation of the effect of cube texture on formability of face center cubic sheet metals, Mecánica Computacional XXVII (2008) 899-907
12. T. Fulop, W.A.M. Brekelmans, M.G.D. Geers, Size effects from grain statistics in ultra-thin metal sheets, J. Mater. Process. Technol 174 (2006) 233-238

13. Landolt - Bornstein Numerical Data and Functional Relationships in Science and Technology. New Series. Group III: Crystal and Solid State Physics. Volume I: Elastic, Piezoelectric and Electrooptic Constants of Crystals, Springer, Berlin, Germany, 1966

14. S. Murakami, Anisotropic aspects of material damage and application of continuum damage mechanics, in CISM Courses and Lectures No. 295, edited by D. Krajcinovic and J. Lemaitre. Springer-Verlag, Wien, New York, 1987, 91-133

15. L.M. Kachanov, Introduction to Continuum Damage Mechanics, Springer-Verlag, 1986

16. Z. Gronostajski, The constitutive equations for FEM, J. Mater. Process. Technol. 106 (2000) 40-44

Cite this article as: Anil Mashalkar, Vilas Nandedkar, Effect of crystallographic texture on the forming limit in microforming of brass, Manufacturing Rev. 6, 27 (2019) 